Supporting Information

\title{
Hierarchical \\ Wrinkle-Structured \\ Catalyst
}

\section{Layer/Membrane Interface for Ultralow Pt-loading}

Polymer Electrolyte Membrane Fuel Cells

\section{(PEMFCs)}

Dong-Hyun Lee ${ }^{\dagger, 1}$, Geun-Tae Yun ${ }^{\dagger, 1}$, Gisu Doo ${ }^{1}$, Seongmin Yuk ${ }^{1}$, Hwanuk Guim², Yesol Kim ${ }^{1}$, Woo-Bin Jung ${ }^{*, 3}$, Hee-Tae Jung ${ }^{*}, 1$, and Hee-Tak Kim ${ }^{*}, 1,4$

${ }^{1}$ Department of Chemical \& Biomolecular Engineering, Korea Advanced Institute of Science and Technology (KAIST), 291, Daehak-ro, Yuseong-gu, Daejeon, 34141, Republic of Korea

${ }^{2}$ Division of Electron Microscopic Research, Korea Basic Science Institute, Daejeon, 34133, Republic of Korea

${ }^{3}$ John A. Paulson School of Engineering and Applied Sciences, Harvard University, Cambridge, MA 02138, USA

${ }^{4}$ Advanced Battery Center, KAIST Institute for the NanoCentury, Korea Advanced Institute of Science and Technology (KAIST), 291, Daehak-ro, Yuseong-gu, Daejeon, 34141, Republic of Korea

This file contains the following information:

Supplementary figures and texts. 


\section{Table of Contents}

\section{EXPERIMENTAL SECTION}

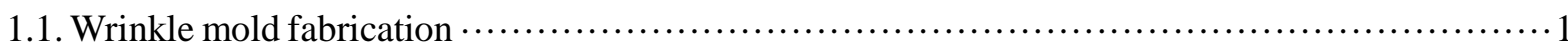

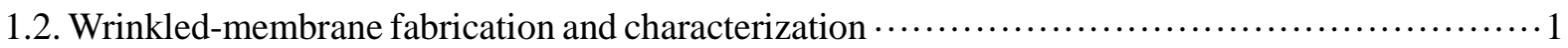

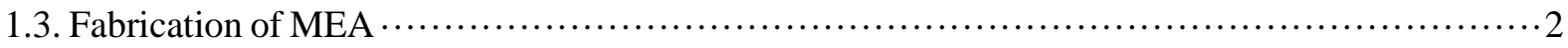

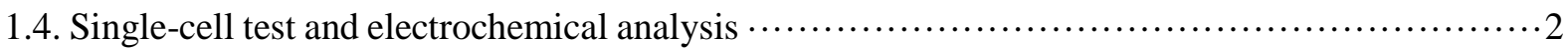

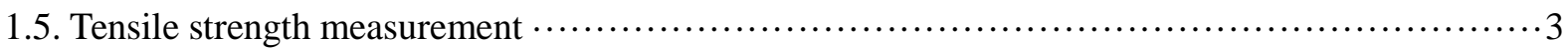

\section{FIGURES}

S1. Morphologies of wrinkled Nafion membrane by using different molds $\cdots \cdots \cdots \cdots \cdots \cdots \cdots \cdots \cdots \cdots \cdots \cdots \cdots$

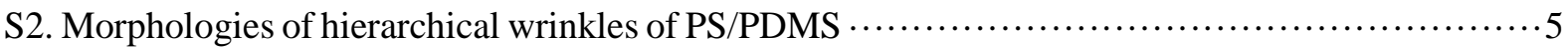

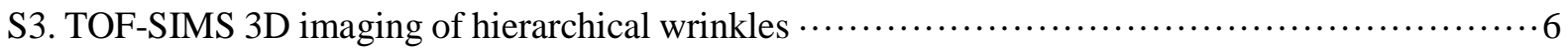

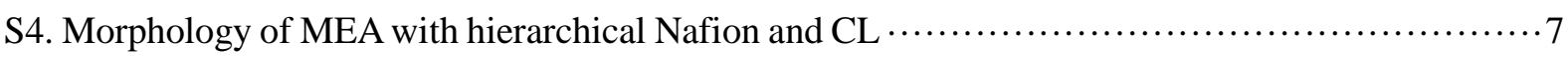

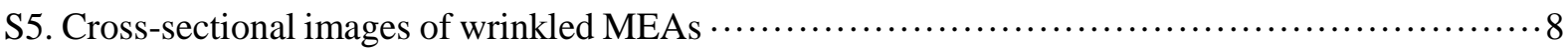

S6. High-frequency resistance measurements and IR-corrected polarization curves of MEAs $\cdots \cdots \cdots \cdots \cdot 9$

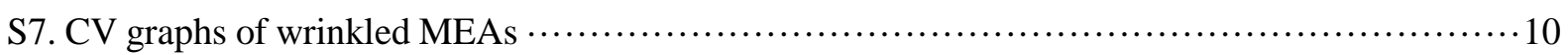

S8. Dynamic light scattering graph of Pt/C and Nafion ionomer agglomerates $\cdots \cdots \cdots \cdots \cdots \cdots \cdots \cdots \cdots \cdots \cdots \cdots \cdots \cdots$

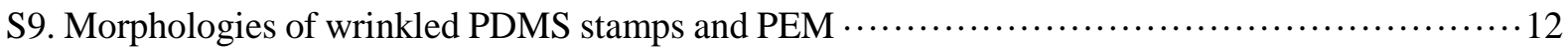

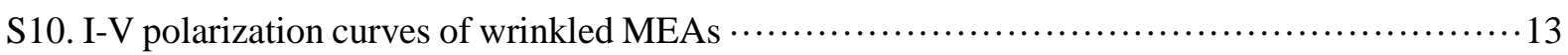

S11. Tafel plots from IR-corrected polarization curves of various MEAs $\cdots \cdots \cdots \cdots \cdots \cdots \cdots \cdots \cdots \cdots \cdots \cdots \cdots \cdots \cdots \cdots \cdots \cdots$

S12. CV graphs and hydrogen desorption peaks of various MEAs $\cdots \cdots \cdots \cdots \cdots \cdots \cdots \cdots \cdots \cdots \cdots \cdots \cdots \cdots \cdots \cdots \cdots \cdots \cdots \cdots \cdots \cdots \cdots$

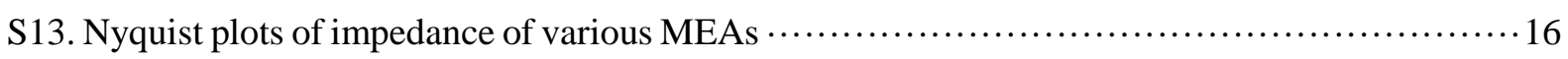

S14. Oxygen transport resistances of F- and G123-75-MEA in He environment $\cdots \cdots \cdots \cdots \cdots \cdots \cdots \cdots \cdots \cdots \cdots \cdots \cdots$

S15. I-V polarization and power density curves of F- and G123-75-MEA $\cdots \cdots \cdots \cdots \cdots \cdots \cdots \cdots \cdots \cdots \cdots \cdots \cdots \cdots \cdots \cdots \cdots$

\section{REFERENCES}




\section{EXPERIMENTAL SECTION}

1.1. Wrinkle mold preparation: We prepared a PS shrinkage film (Grafix, KSF50-C) with a thickness of $250 \mu \mathrm{m}$ and a $T_{g}$ of $120^{\circ} \mathrm{C}$ as a template. The PS film was treated with $\mathrm{Ar}^{+}$plasma to generate the skin layer. Afterward, the sample was heated in an oven above $130^{\circ} \mathrm{C}$, which resulted in the formation of G1 wrinkles on the entire surface. To fabricate microscale G2 and G3 wrinkles, we coated the surface with polyvinyl pyrrolidone (PVP, $\mathrm{M}_{\mathrm{w}}=360000 \mathrm{gmol}^{-1}, \mathrm{~T}_{\mathrm{g}}$ $=150^{\circ} \mathrm{C}$ ) as a sacrificial polymer skin layer; it was quickly removed with ethanol. After coating the polymer layer onto a PS film with G1 wrinkles, we repeated the heating and cooling processes to generate hierarchical wrinkles. To generate wrinkles with larger wavelengths, we used a sacrificial skin layer with a greater thickness. We poured PDMS with a controlled base elastomer-to-curing agent ratio of 5:1 (Sylgard 184, Sigma Aldrich) onto a PS film to create a stamp. The PDMS mold was cured for $4 \mathrm{~h}$, which resulted in the reverse image of the hierarchical wrinkles on the PS film. In addition, a model system with 2D gold wrinkles was prepared with the same wrinkling process; a gold layer (40 nm) was deposited onto a PS substrate via e-beam evaporation.

1.2. Wrinkled-membrane fabrication and characterization: The wrinkled structures on the PDMS molds were transferred onto a Nafion 211 membrane (25 $\mu$ m-thick, Dupont) with the hot-pressing method. The membrane was sandwiched between an anode CL and the wrinkled PDMS mold and pressed at $130{ }^{\circ} \mathrm{C}$. The lamination time (30 min) and pressure (20 atm) were carefully chosen to increase the quality of the transferred pattern and prevent dimensional changes in the Nafion membrane. Moreover, elastic silicone rubber films were located onto the fronts and backs of the stamp and Nafion membrane stack as pressure distribution layers. After heat lamination, the fluorinated ethylene propylene films on the anode CL and wrinkled PDMS 
mold were removed from the wrinkled PEM. The surface morphologies of the wrinkled PEM and MEA were studied with a field emission-scanning electron microscope (Sirion, FEI); in addition, 3D surface images were recorded with time-of-flight secondary ion mass spectrometry (ION-TOF GmbH, TOF-SIMS5).

1.3. Fabrication of MEA: The CL was coated onto the wrinkled membrane via spray coating. The slurry ink was composed of Pt/C (TEC10F50E, Tanaka Kikinzoku Kogyo), a Nafion ionomer dispersion (D520, Dupont), and additional binary solvents (deionized water and 1propanol in a 1:1 weight ratio). The solid content in the slurry ink was $3 \%$ by weight, and the ionomer-to-carbon weight ratio was $0.7: 1$. The mixture was homogeneously dispersed via tip sonication for $90 \mathrm{~min}$. The spray coating process was conducted with a handheld spray gun on a $60^{\circ} \mathrm{C}$-heated plate. The active area of the catalyst-coated membrane (CCM) in the projected area was $12.25 \mathrm{~cm}^{2}$, and the cathode $L_{p t}$ was $0.05,0.1$, and $0.2 \mathrm{mg}_{\mathrm{Pt}} \mathrm{cm}^{-2}$. As the counterpart of cathode, the anode Pt-loading was fixed as $0.2 \mathrm{mg} / \mathrm{cm}^{2}$ to remove polarization.

1.4. Single-cell test and electrochemical analysis: A single cell was assembled with a CCM; a pair of gas diffusion layers (39BC, SGL Carbon), pair of hard gaskets, and pair of graphite blocks created a single serpentine flow field between the endplates. The I-V polarization curves and HFR were measured with a fuel cell station (Scitech Korea) at $65^{\circ} \mathrm{C}$ at an absolute pressure of $180 \mathrm{kPa}$ and $30 \% \mathrm{RH}$. The operation temperature was chosen as the realistic operation condition of fuel cell-based vehicles. Moreover, the stoichiometry of the feed gases was 1.2 for hydrogen and 1.5 for air/oxygen. The minimal flow rates were $46 \mathrm{sccm}$ for hydrogen and 136 sccm for air/oxygen, which is equivalent to generate $2 \mathrm{~A}$. The electrochemical characterization, EIS and CV measurements were conducted with a potentiostat (HCP-803, BioLogics Science Instruments). The EIS measurements were performed at 0.15 and $0.5 \mathrm{Acm}^{-2}$ while feed gas was 
supplied to the anode and cathode; the frequency range was $100 \mathrm{kHz}$ to $0.1 \mathrm{~Hz}$. Furthermore, the $\mathrm{CV}$ measurement was conducted at $0.069-1.2 \mathrm{~V}$ at a hydrogen flow of $100 \mathrm{sccm}$ supplied to the anode and $50 \mathrm{mV}$ scan rate. The experimental details of the limiting current method were adopted from previous studies. ${ }^{1,2}$ The excess hydrogen (500 sccm) and diluted oxygen (650 sccm, $1 \%$ oxygen in helium or nitrogen) amounts were injected into the anode and cathode with $1 \mathrm{~cm}^{2}$ active areas. Finally, the linear sweep voltammetry measurements were performed at an OCV of $0.15 \mathrm{~V}$ with $5 \mathrm{mVs}^{-1}$ scan rate at different cell temperatures $\left(50,65\right.$, and $\left.80{ }^{\circ} \mathrm{C}\right)$.

1.5. Tensile strength measurement: The tensile strength of Nafion membranes were measured via universal testing machine (UTM) (T150, KLA). One end of specimens (bare and hierarchical-wrinkled Nafion films) was fixed in the upper cross-head of the machine. In addition, the other end of specimens was fixed at the movable cross-head. After loading, tensile load is gradually applied to the specimen until the specimen tears. As a result, the tensile strength measurement was achieved as a graph of tensile stress and strain. 


\section{FIGURES}
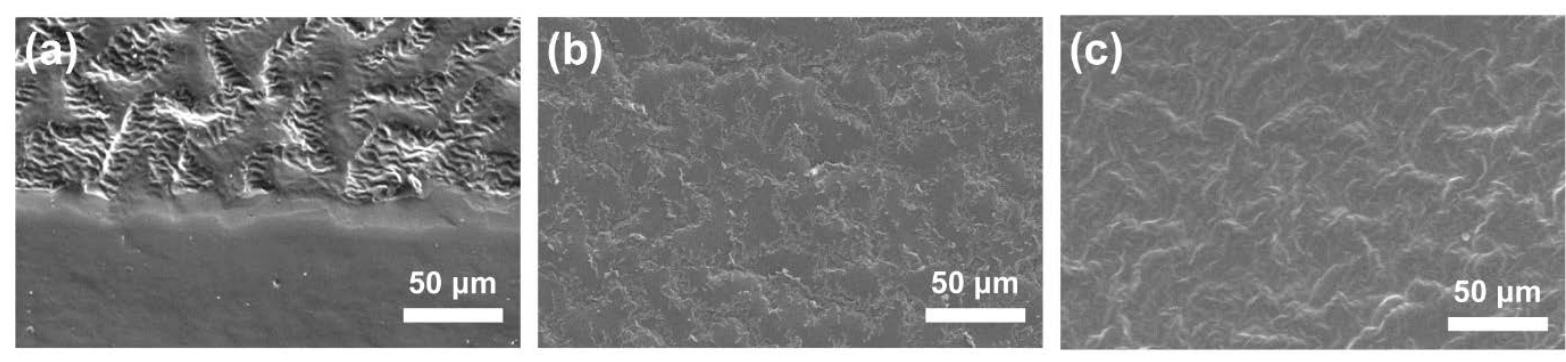

Figure S1. Morphologies of wrinkled Nafion membrane by using different molds. SEM images of imprinted Nafion membrane with (a) PDMS, (b) NOA, and (c) PDMS/rubber compound (5:1 weight ratio) molds. 

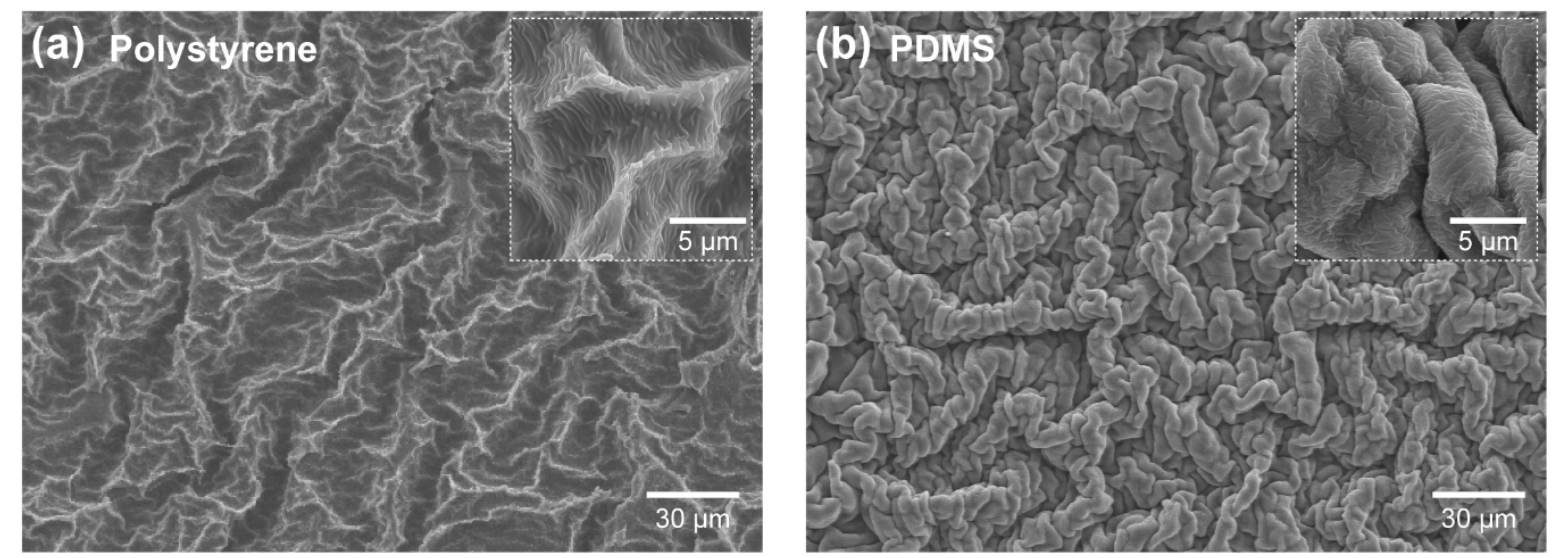

Figure S2. Morphologies of hierarchical wrinkles of PS/PDMS. SEM images of a G123-75

(a) PS substrate and (b) PDMS stamp. 


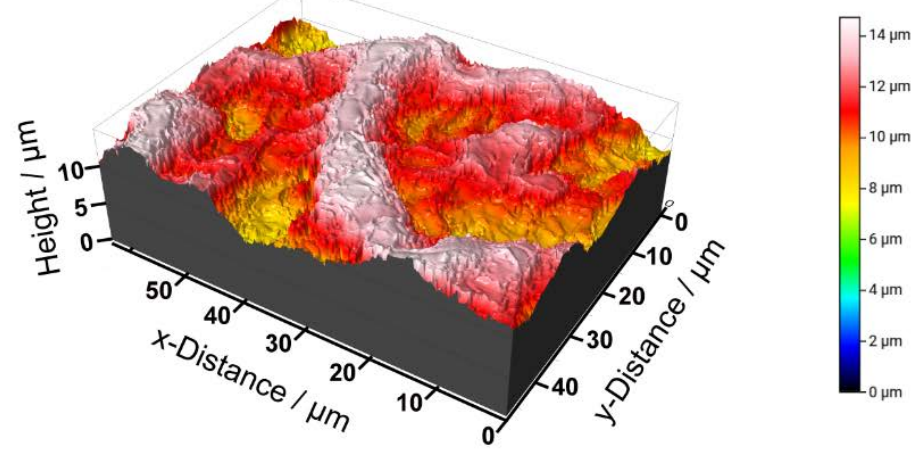

Figure S3. TOF-SIMS 3D imaging of hierarchical wrinkles. Image of G123-75 hierarchical wrinkle-structured membrane obtained with TOF-SIMS 3D imaging. 


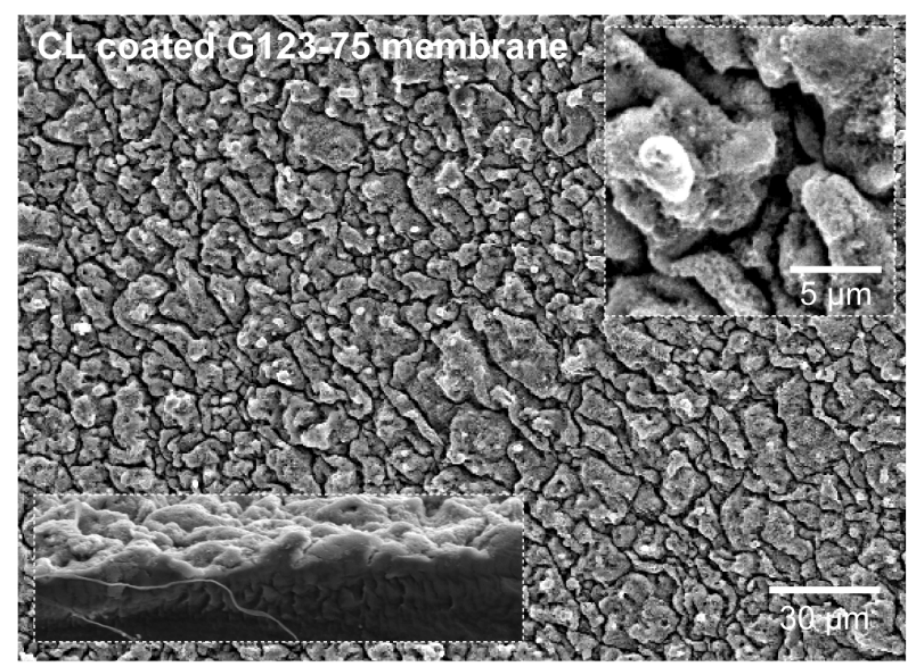

Figure S4. Morphology of MEA with hierarchical Nafion and CL. Top-view SEM images of G123-75-MEA. 

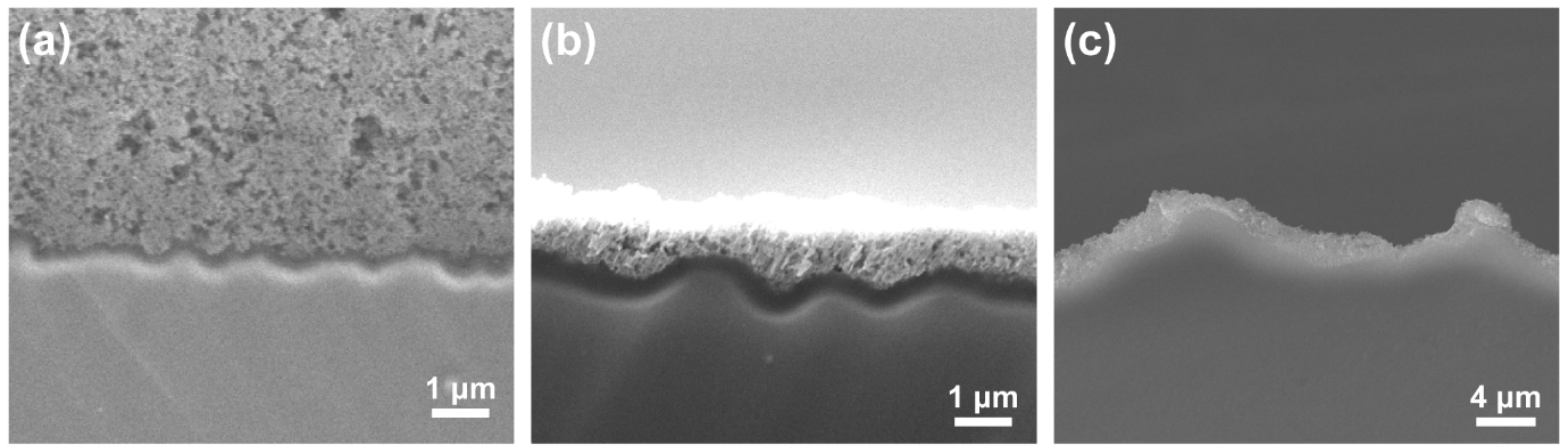

Figure S5. Cross-sectional images of wrinkled MEAs. Cross-sectional SEM images of (a) G1-37-MEA, (b) G2-37-MEA, and (c) G3-37-MEA. 

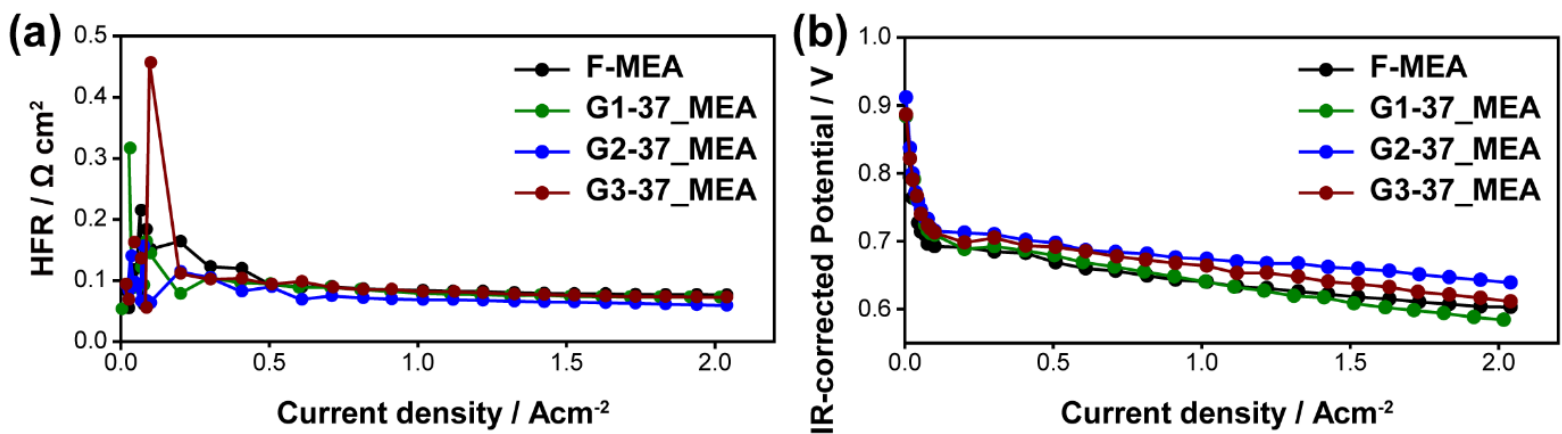

Figure S6. High-frequency resistance measurements and IR-corrected polarization curves of MEAs. (a) High-frequency resistance (HFR) measurements and (b) IR-corrected polarization curves of F-MEA, G1-37-MEA, G2-37-MEA, and G3-37-MEA. The experiments were conducted in an oxygen environment to prevent mass transport resistance. 


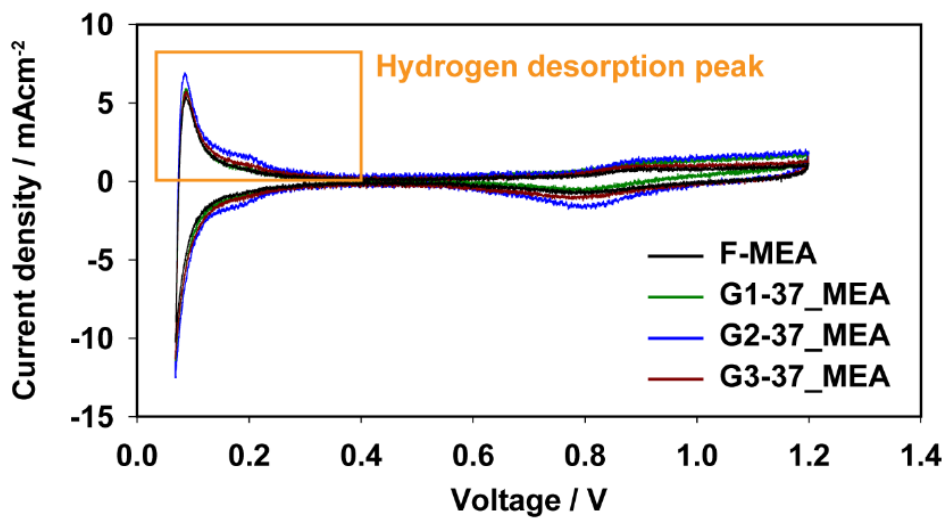

Figure S7. CV graphs of wrinkled MEAs. CV graphs of F-, G1-37-, G2-37-, and G3-37MEA. 


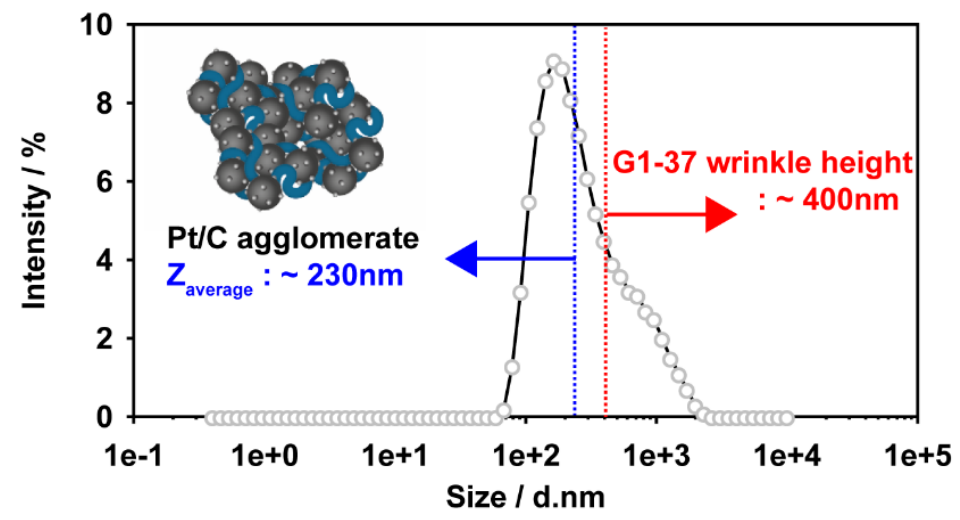

Figure S8. Dynamic light scattering graph of Pt/C and Nafion ionomer agglomerates.

Dynamic light scattering graph of Pt/C and Nafion ionomer agglomerates in water/IPA solvent mixture (1:1 weight ratio). 

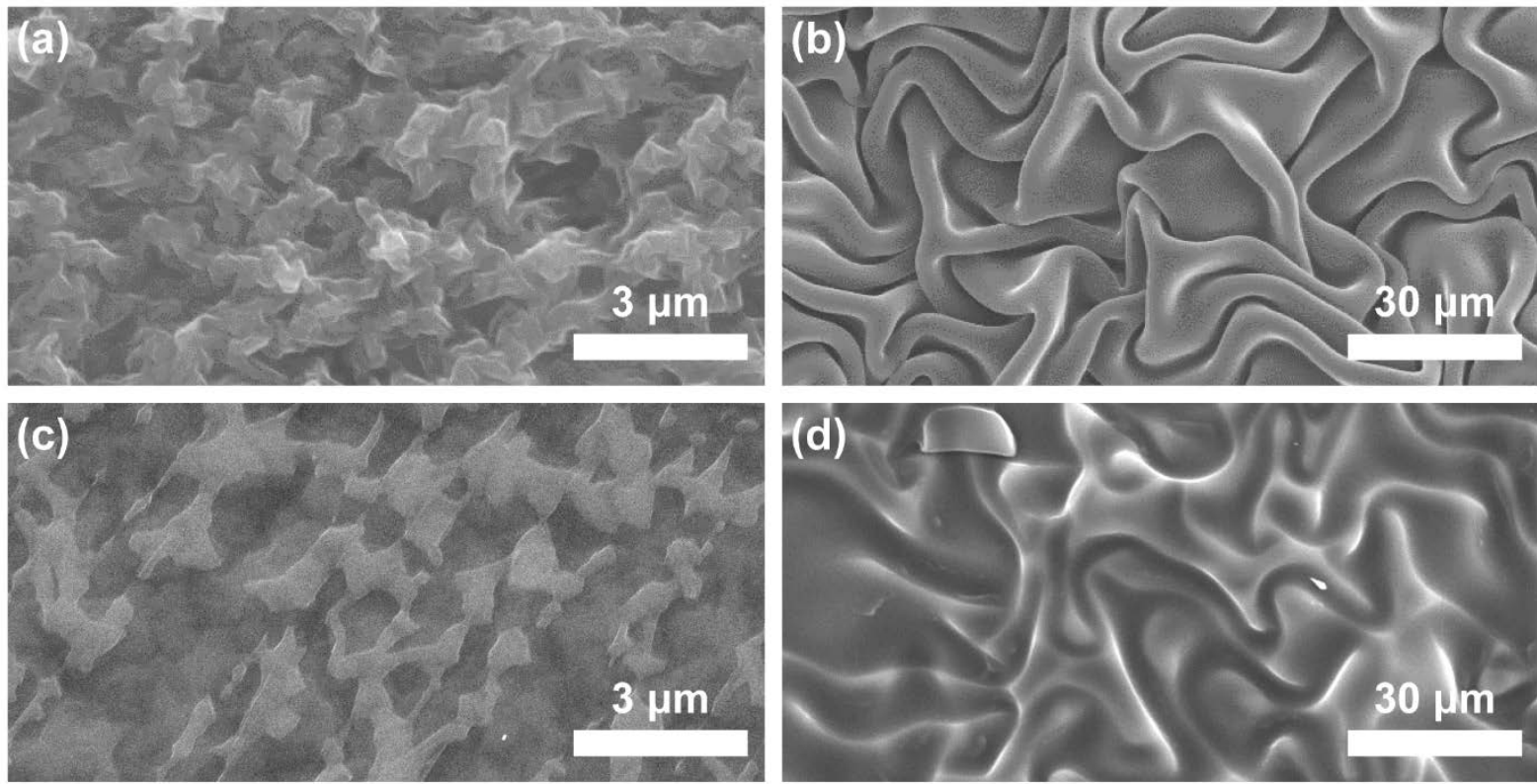

Figure S9. Morphologies of wrinkled PDMS stamps and PEM. Comparison of top-view

SEM images for PDMS stamps of (a) G1-75, (b) G3-75 and corresponding PEM of (c) G1-75, (d) G2-75. 
(a)

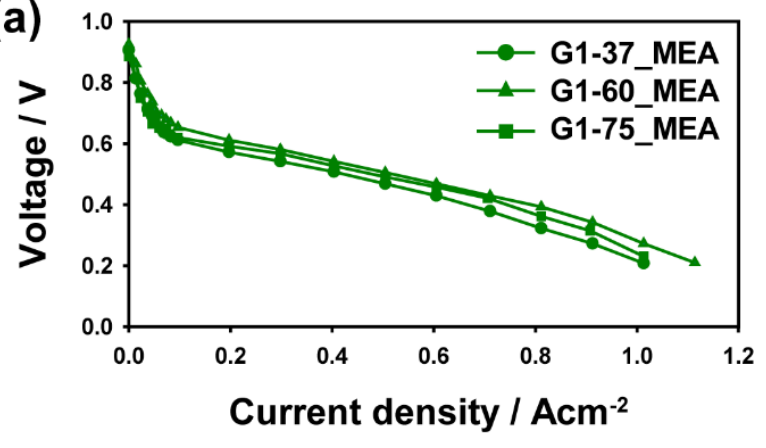

(b)

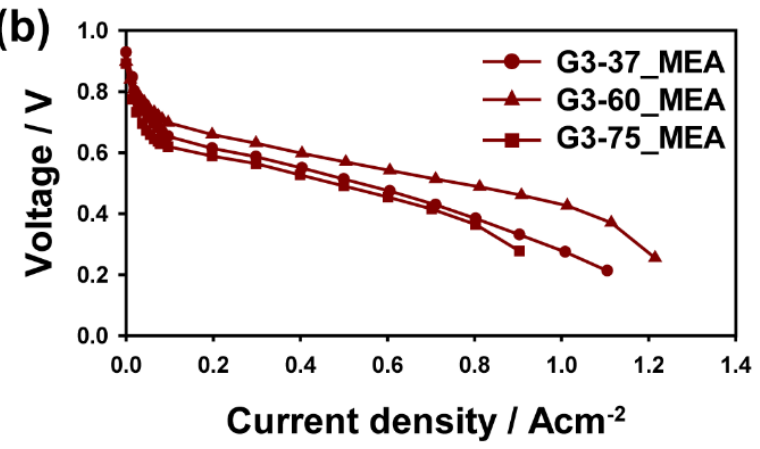

Figure S10. I-V polarization curves of wrinkled MEAs. I-V polarization curves of (a) G1MEA and (b) G3-MEA with $\varepsilon=0.37,0.6$, and 0.75 . 


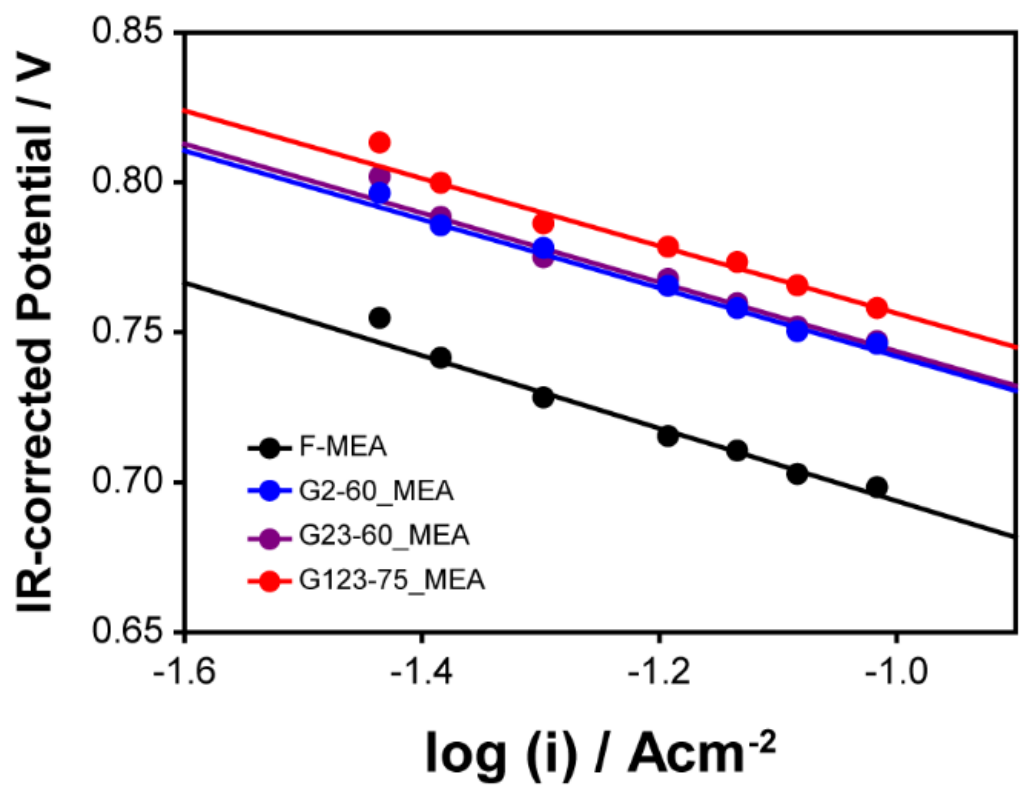

Figure S11. Tafel plots from IR-corrected polarization curves of various MEAs. Tafel plots produced from IR-corrected polarization curves of F-, G2-60-, G23-60-, and G123-75-MEA measured with oxygen. 


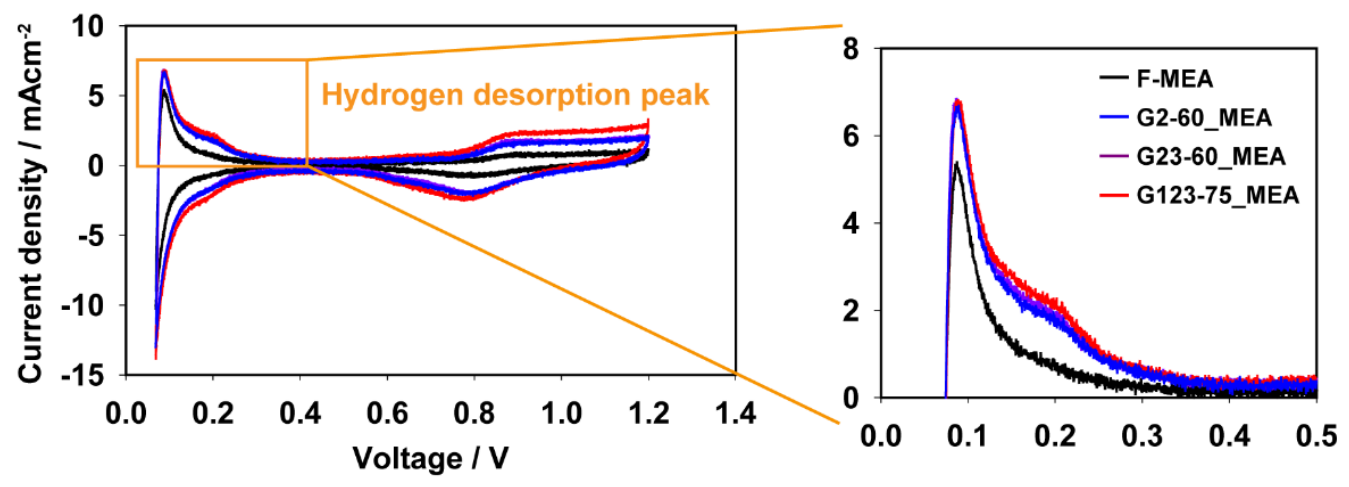

Figure S12. CV graphs and hydrogen desorption peaks of various MEAs. CV graphs and enlarged hydrogen desorption peaks of F-, G2-60-, G23-60-, and G123-75-MEA. 

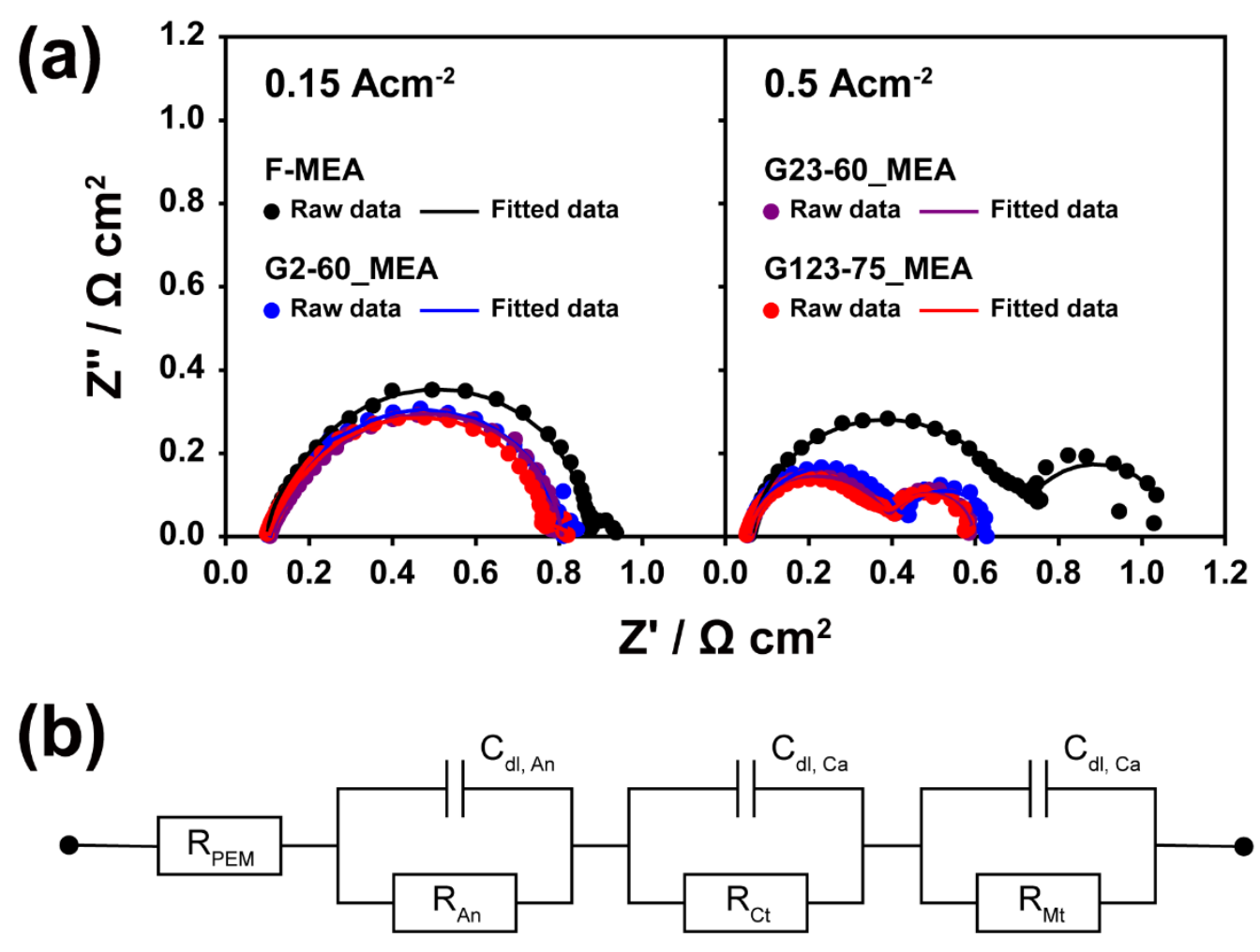

Figure S13. Nyquist plots of impedance of various MEAs. (a) Nyquist plots of impedance of F-, G2-60-, G23-60-, and G123-75-MEA at 0.15 and $0.5 \mathrm{~A} \mathrm{~cm}^{-2}$ and (b) equivalent circuit model for Nyquist plot fit. 
Oxygen transport resistances in He environment

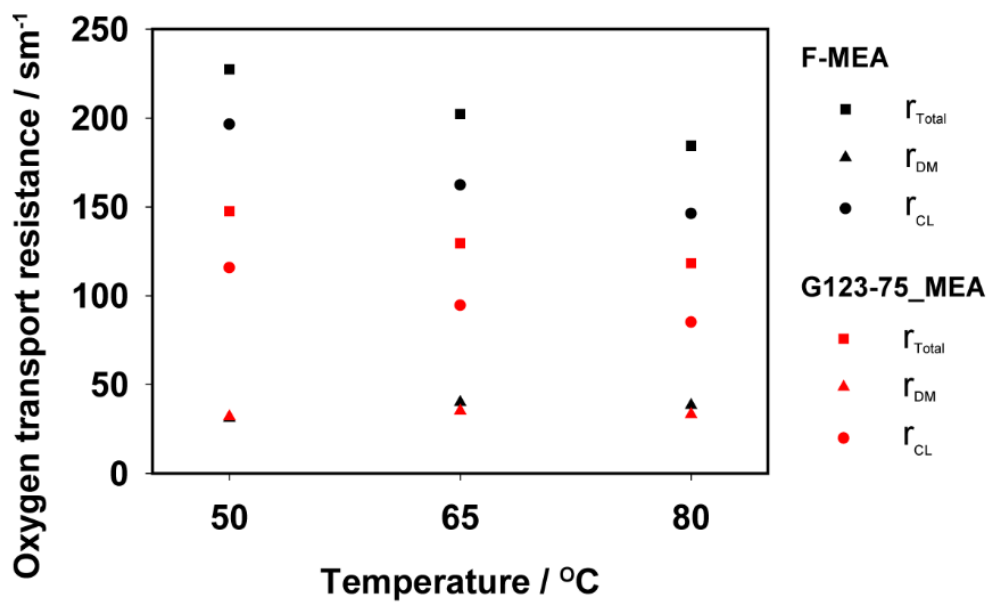

Figure S14. Oxygen transport resistances of F- and G123-75-MEA in He environment 


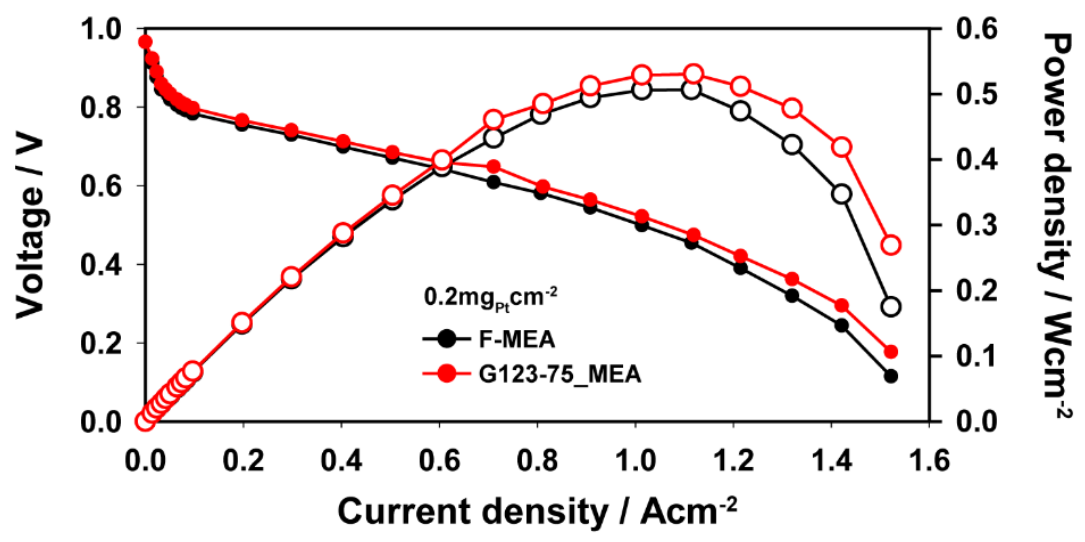

Figure S15. I-V polarization and power density curves of F- and G123-75-MEA 


\section{REFERENCES}

(1) D.-H. Lee.; W. Jo.; S. Yuk.; J. Choi.; S. Choi.; G. Doo.; D. W. Lee.; H.-T. Kim. In-Plane Channel-Structured Catalyst Layer for Polymer Electrolyte Membrane Fuel Cells. ACS Appl. Mater. Interfaces 2018, 10, 4682-4688.

(2) D.-H. Lee.; M. K. Kim.; H. Guim.; S. Yuk.; J. Choi.; S. Choi.; G. Doo.; D. W. Lee.; J. Noh.; H.-T. Kim. Ditch-structured microprous layers fabricated by nanosecond-pulse laser ablation for enhancing water transport in polymer electrolyte membrane fuel cells. Mater. Adv. 2020, 1, 254-261. 